\title{
Penetration of the Descending Colon by a Migrating Intrauterine Contraceptive Device
}

\author{
Jung Min Park, Chang Seog Lee, Min Seong Kim, Do Young Kim, Chul Young Kim, Young Bae Lim, \\ Yong Kyu Lee, Dong Eun Park ${ }^{1}$, Dong Hyun Lee \\ Departments of Internal Medicine and ${ }^{1}$ Surgery, Good Gang-An Hospital, Busan, Korea
}

Foreign bodies in the gastrointestinal tract often cause serious complications, such as perforation, obstruction, abscess formation, or hemorrhage. This is a case in which a patient visited our hospital and complained of a vague lower abdominal pain that had been present for three months. She had an intrauterine device (IUD) inserted five years earlier. The abdominal X-ray, computed tomography and colonoscopy revealed that the IUD had penetrated into the descending colon. We tried to remove the IUD by colonoscopy but failed due to pain, so we removed the IUD surgically. Thus, we report a case in which a previously inserted IUD had penetrated into the descending colon and was surgically removed. We also present a brief review of the literature.

Keywords: Foreign bodies; Intrauterine contraceptive device; Colon perforation

\section{INTRODUCTION}

In comparison with the upper gastrointestinal tract (UGI), reports on foreign bodies in the low gastrointestinal tract (LGI) are rare because patients cannot recognize the presence of a foreign body in many cases and because the symptoms of a foreign body in the LGI are vague and diverse. If a foreign body in the intestine is not excreted spontaneously, and acute abdomen, such as intestinal perforation, peritonitis, etc., may be shown [1]. Generally, foreign bodies detected in the LGI are swallowed, transit the intestinal tract, are retained within the intestinal tract and are, thus, detected in many cases; nevertheless, depending on the size and the shape of foreign body, the materials, and the characteristics of the patient, the foreign body may be detected in an unexpected area or pathway [2].

We experienced a case in which an intrauterine contraceptive

Received: July 24, 2010 Accepted: September 23, 2010

Correspondence to: Dong Hyun Lee, M.D.

Department of Internal Medicine, Good Gang-An Hospital,

40-1 Namcheon-dong, Suyeong-gu, Busan 613-010, Korea

Tel: +82-51-610-9010, Fax: +82-51-621-1500

E-mail:doongpa@gmail.com

(C) 2010 The Korean Society of Coloproctology

This is an open-access article distributed under the terms of the Creative Commons Attribution NonCommercial License (http://creativecommons.org/licenses/by-nc/3.0) which permits unrestricted noncommercial use, distribution, and reproduction in any medium, provided the original work is properly cited. device (IUD) was detected in the descending colon by abdominal X-ray, abdominal computed tomography, and colonoscopy and was treated by surgically by using a laparatomy. That case is reported together with a review of the literature.

\section{CASE REPORT}

A 42-year-old female patient was admitted for pain in the lower abdomen that had started three months earlier. She had no special past history or social history and had been pregnant four times and delivered twice. Five years earlier, an IUD had been inserted at a private clinic, and no special symptoms were detected. From 3 months prior to admission, she had complained of vague lower left abdominal pain. In regard to vital signs at the time of admission, her blood pressure was 120/80 $\mathrm{mmHg}$, her pulse was 70 times/minute, her respiration rate was 20 times/ minute, and her body temperature was $36.6^{\circ} \mathrm{C}$. She was conscious, with injected conjunctiva, icteric sclera, a skin rash, an oral ulcer, and dehydration of the tongue; no other abnormal findings were detected. In abdominal examination, hepatomegaly and splenomegaly were not detected, and the bowel sound was normal. Tenderness was felt in the lower left abdominal area; nonetheless, rebound tenderness was not detected. In the musculoskeletal system, abnormal findings were not detected. On the peripheral blood test, leucocytes were $10,000 / \mathrm{mm}^{3}$ (neurtophils, $83 \%$ ), hemoglobin was $13.1 \mathrm{~g} / \mathrm{dL}$, and hematocrits were 
Journal of The Korean Society of Penetration of the Descending Colon by a Migrating Intrauterine Contraceptive Device

Coloproctology Jung Min Park, et al.

$39.3 \%$. On the serum biochemistry test, total protein was 7.5 $\mathrm{g} / \mathrm{dL}$, albumin was $4.4 \mathrm{~g} / \mathrm{dL}$, serum cholesterol was $232 \mathrm{mg} / \mathrm{dL}$, AST was $15 \mathrm{U} / \mathrm{L}$, ALT was $13 \mathrm{U} / \mathrm{L}, \mathrm{LDH}$ was $347 \mathrm{U} / \mathrm{L}$, ALP was $130 \mathrm{U} / \mathrm{L}, \mathrm{r}-\mathrm{GTP}$ was $21 \mathrm{U} / \mathrm{L}, \mathrm{Na}$ was $138 \mathrm{mEq} / \mathrm{L}$, and $\mathrm{K}$ was 3.4 $\mathrm{mEq} / \mathrm{L}$. During radiological tests, a foreign body in the abdominal cavity was detected by simple abdominal X-ray (Fig. 1). In abdominal computed tomography, the presence of a V-shaped substance in the vicinity of the descending colon was observed (Fig. 2). Subsequently, to confirm and remove the foreign body, a colonoscopic test was performed, and in the descending colon, $40 \mathrm{~cm}$ away from the anal verge, an IUD that had penetrated the barrier and protruded into the lumen by approximately $3-4 \mathrm{~cm}$ was detected (Fig. 3).

Endoscopic foreign body removal was attempted, but the IUD could not be removed readily, and the patient presented with severe pain; thus, laparoscopic foreign body removal surgery was performed. While administering prophylactic antibiotics under general anesthesia, a laparotomy was performed by us-

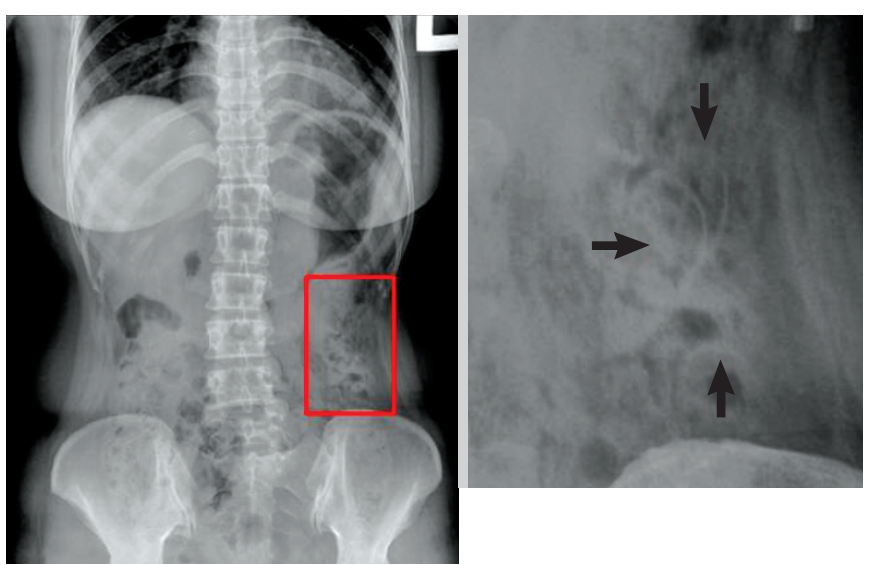

Fig. 1. Plain abdominal X-ray. A misplaced intrauterine device in the left lower quadrant area.
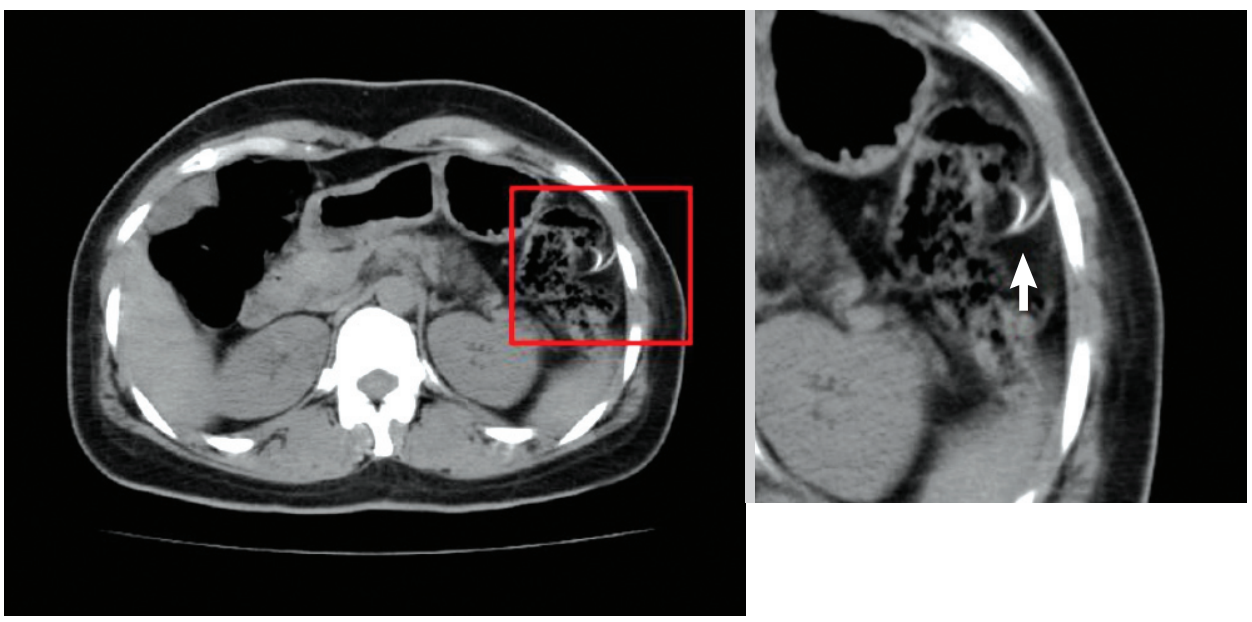
into the lumen of the descending colon.
Fig. 3. Colonscopic finding. The intrauterine device had penetrated

ing the perimidline incision. When the adhered omentum was resected, an IUD (ParaGard T 380A; DuraMed, Pomona, NY, USA) that had penetrated the large intestine wall was detected in the descending colon. A trace of fibrosis in the uterine fundus was observed; nonetheless, special findings in other abdominal areas and pelvic organs were not detected. Thus, the foreign body was removed by using a transverse resection of the area adjacent to the colon where the foreign body had, and the intestine suture procedure was performed (Fig. 4). After surgery, the patient did not have special abnormal findings or complications and was discharged 8 days after admission.

\section{DISCUSSION}

Not only is penetration of a foreign body into the large intestinal tract rare, its symptoms are vague and diverse; thus, its clinical diagnosis may be delayed, and it may be detected incidentally by radiological tests. For its diagnosis, the symptoms of the

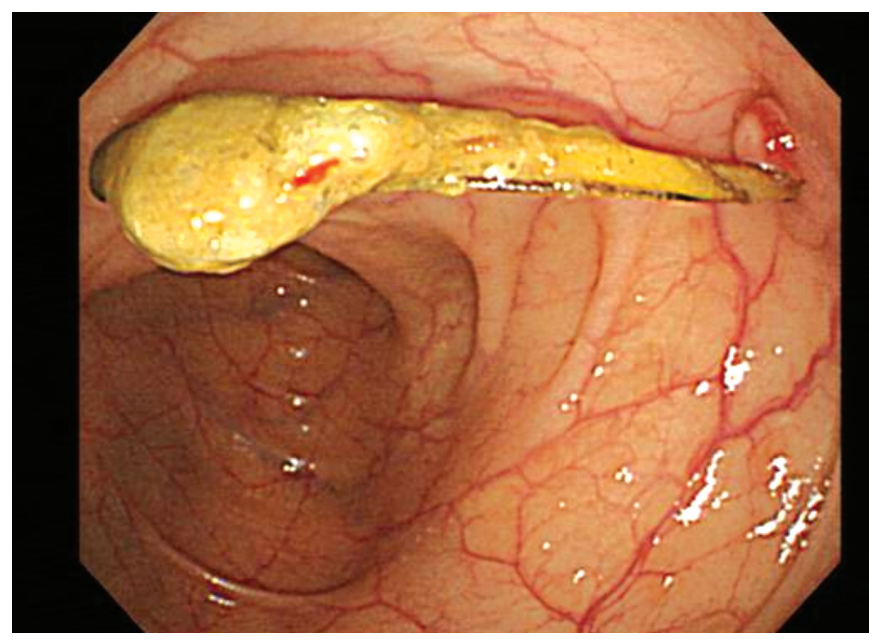

Fig. 2. Abdominal computed tomography scan. The intrauterine device is embedded adjacent to the descending colon. 


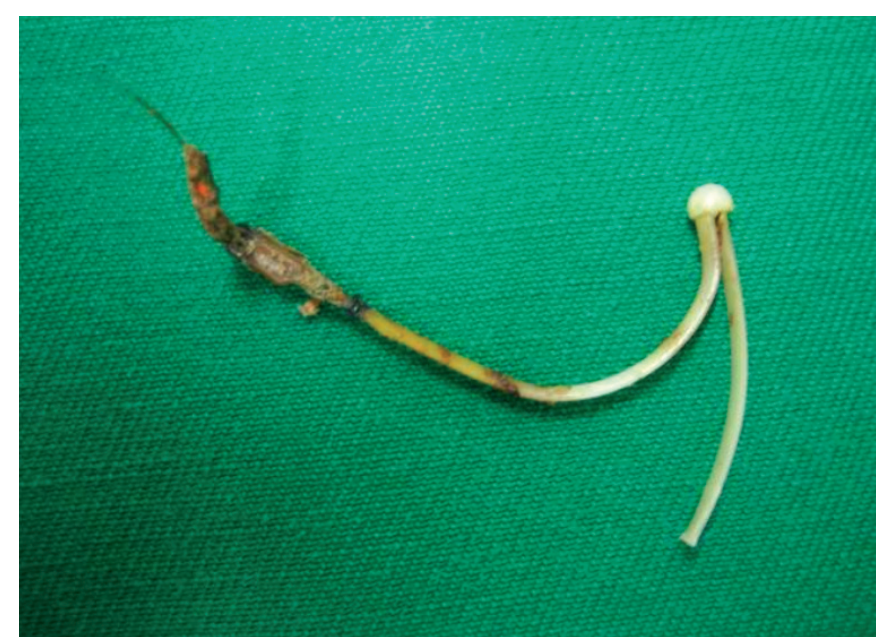

Fig. 4. Gross finding. The intrauterine device that had been removed from the descending colon.

patients and the past history of exposure to foreign bodies are important, and the type and the properties of the foreign body, the length of the foreign body, the interval since exposure, and the medical, as well as surgical, disease history should be considered. Occasionally, the diagnosis of a foreign body in the LGI, as well as treatment, is delayed; thus, if compression necrosis of the intestinal wall progresses, an intestinal perforation, an intestinal fistula, or an abscess within the abdominal cavity may form. In addition, in cases in which the foreign object is fixed in a specific area and spontaneous expel is delayed, the possibility of developing complications is high, so it should be removed immediately, regardless of symptoms $[1,2]$.

The IUD is simple and safe and can be used effectively without spontaneous expulsion so that continuous contraception is obtained; hence, it is used by approximately 160,000,000 women worldwide. The IUD has been associated with such complications as hemorrhage, pain, pregnancy, spontaneous expulsion, uterine perforation, infection, and translocation in adjacent organs [3]. Migration of an IUD from the uterus to other organs can be considered to be the result of expulsion of the IUD and uterine perforation. The expulsion of the device is a complication reported rarely, and risk factors are past history of expulsion, dysmenorrhea, and young age. Diverse symptoms from asymptomatic to vaginal bleeding, pelvic pain, and dyspareunia may be shown, and if the string of the device is not shown, it must be differentiated by using simple abdominal imaging and vaginal ultrasonography [4].

Uterine perforation by an IUD is as rare as 0.87 in 1,000 person; nonetheless, it is one of most dangerous complications [5, $6]$, and it may invade adjacent organs, the small intestine, the sigmoid colon, the cecum, and the appendix. Although the incidence of uterine perforation and translocation within the abdominal cavity is low, it is a serious complication that has been reported in Korea several times. Cases of an IUD in the sigmoid colon have been reported. Our patient, however, had an IUD that had perforated the descending colon, and until now, such cases have not been reported [7].

If a foreign body is fixated in the intestine without changing, its endoscopic removal should be attempted, and if such methods fail, surgical removal should be performed. In our case, similarly, attempts were made to remove the IUD by using colonoscopic procedures, but the patient presented with severe pain; thus, the IUD was removed by a resection of the colon. In cases with a foreign body within the intestinal tract for which the symptoms are similar to those of an acute abdomen and in cases associated with complications such as peritonitis, abscess, and fistula caused by intestinal perforation, the general method is to remove the foreign body by using a surgical laparotomy and to treat complications. Particularly, for cases showing abdominal pain, fever, intermittent diarrhea, considering the damage to the intestines, it must be removed $[1,2,7,8]$. Therefore, if patients with a past history of an IUD develop symptoms of vague pain in the abdomen, vaginal hemorrhage, and dyspareunia, considering the possibility of expulsion of the device or uterine perforation, in addition to the basic history taking and physical examination, aggressive diagnosis by imaging medical tests is required.

In conclusion, foreign bodies within the gastrointestinal tract are spontaneously expelled in most cases; nonetheless, if sharp and hard substances are fixated in the intestinal tract and are not expelled, thus inducing diverse complications, it is best to remove them rapidly. If the initial removal of the foreign bodies immobilized in the intestine fails, surgical removal should be performed prior to the development of other complications.

\section{CONFLICT OF INTEREST}

No potential conflict of interest relevant to this article was reported.

\section{REFERENCES}

1. Park JI, Eum SH, Kim CY, Yoon JH, Hwang HS, Kim YS, et al. A case of colonoscopically removed toothpick in the sigmoid colon. Korean J Med 2006;71:668-72.

2. Lee EJ, Kim HJ, Jung KA, Lim RR, Kim TH, Lee OJ, et al. Endodontic file in an appendix. Korean J Med 2007;72:209-12.

3. Barwin BN, Tuttle S, Jolly EE. The intrauterine contraceptive device. Can Med Assoc J 1978;118:53-8.

4. Carmody K, Schwartz B, Chang A. Extrauterine migration of a Mirena(R) intrauterine device: a case report. J Emerg Med 2010 [Epub]. DOI:10.1016/j.jemermed.2010.04.024.

5. Choi JH, Suh JM, Lee HJ, Oh JH, Lee GS, Hur SY, et al. Misplaced IUD. Korean J Obstet Gynecol 2004;47:1960-4.

6. Arslan A, Kanat-Pektas M, Yesilyurt H, Bilge U. Colon penetra- 


\section{Coloproctology Jung Min Park, et al.}

tion by a copper intrauterine device: a case report with literature review. Arch Gynecol Obstet 2009;279:395-7.

7. Pirwany IR, Boddy K. Colocolic fistula caused by a previously inserted intrauterine device: case report. Contraception 1997;
56:337-9.

8. Kim SY, Park CM. A case of displacement of intrauterine device into abdominal cavity by uterine perforation in early pregnancy. Korean J Obstet Gynecol 2008;51:256-60. 